\title{
LOCAL INVERTIBILITY IN SUBRINGS OF $C^{*}(X)$
}

\author{
H. Linda Byun, Lothar Redlin and Saleem Watson
}

\begin{abstract}
It is known that the maximal ideals in the rings $C(X)$ and $C^{*}(X)$ of continuous and bounded continuous functions on $X$, respectively, are in one-to-one correspondence with $\beta X$. We have shown previously that the same is true for any ring $A(X)$ between $C(X)$ and $C^{*}(X)$. Here we consider the problem for rings $A(X)$ contained in $C^{*}(X)$ which are complete rings of functions (that is, they contain the constants, separate points and closed sets, and are uniformly closed). For every noninvertible $f \in A(X)$, we define a $z$-filter $Z_{A}(f)$ on $X$ which, in a sense, provides a measure of where $f$ is 'locally invertible'. We show that the map $Z_{A}$ generates a correspondence between ideals of $A(X)$ and $z$-filters on $X$. Using this correspondence, we construct a unique compactification of $X$ for every complete ring of functions. Each such compactification is explicitly identified as a quotient of $\beta X$. In fact, every compactification of $X$ arises from some complete ring of functions $A(X)$ via this construction. We also describe the intersections of the free ideals and of the free maximal ideals in complete rings of functions.
\end{abstract}

\section{INTRODUCTION}

If $X$ is a completely regular space then the collection $C(X)$ of continuous realvalued functions on $X$ is a ring under pointwise operations. The ring $C(X)$ and its subring $C^{*}(X)$, consisting of the bounded elements of $C(X)$, have been studied extensively (see [4] and [1]). The resulting theory of 'rings of continuous functions' is beautifully presented in the now classic text of Gillman and Jerison by that title. One of the main achievements in this theory is the characterisation of the maximal ideals in the rings $C(X)$ and $C^{*}(X)$. Although the problems of characterising the maximal ideals in $C(X)$ and $C^{*}(X)$ are quite different, they have the same solution-namely, the set of maximal ideals in each is in one-to-one correspondence with $\beta X$, the Stone-Cech compactification of $X$. In [6], a method was developed to characterise the maximal ideals in any ring $A(X)$ of continuous functions on $X$ with $A(X) \supseteq C^{*}(X)$, and in [2] the method was used to study deeper properties of these rings. The technique of 'local invertibility' used in these papers has the desirable effect of putting the problems of characterising the maximal ideals in $C(X)$ and $C^{*}(X)$ into a common setting. This

Received 22 November 1991

Copyrighi Clearance Centre, Inc. Serial-fee code: 0004-9729/92 \$A2.00+0.00. 
is achieved by defining a map $Z_{A}$ from the noninvertible elements of $A(X)$ to the $z$ filters on $X$, which lifts to one that maps ideals in $A(X)$ to $z$-filters. In this paper we consider how the concept of local invertibility can be applied to rings $A(X)$ contained in $C^{*}(X)$. It turns out that the rings for which an analogous map $Z_{A}$ is useful are complete rings of functions - those which contain the constants, separates points and closed sets, and are uniformly closed. The rings $A(X) \supseteq C^{*}(X)$ studied in [6] and [2] in fact satisfy these conditions; however, the proofs there make essential use of the fact that $A(X) \supseteq C^{*}(X)$ so they do not in general carry over to the case we consider here.

In Section 1 we define the map $Z_{A}$ and show that it maps ideals to $z$-filters. We prove that $Z_{A}^{\leftarrow}$, the inverse of $Z_{A}$, maps $z$-ultrafilters to maximal ideals, although not necessarily in a one-to-one fashion (as opposed to the case in which $C^{*}(X) \subseteq A(X)$ ). In Section 2 we use $Z_{A}^{\leftarrow}$ to construct a correspondence between compactifications of $X$ and complete rings of functions on $X$. (Compare [3, p.301].) Our correspondence is explicit in the sense that a procedure is given for determining those points of $\beta X$ (that is, $z$-ultrafilters on $X$ ) which are identified to yield the compactification that corresponds to any specified $A(X)$. Finally, in Section 3 we study the intersections of the free ideals and the free maximal ideals in $A(X)$. Any terminology or notation not specifically defined in this paper is to be understood as in [4].

\section{The CORRESPONDENCE BETWEen IDEALS AND FILTERS}

Let $X$ be a completely regular space. A subalgebra $A(X)$ of $C^{*}(X)$ is called a complete ring of functions if it contains the constants, separates points and closed sets, and is uniformly closed (see Engelking [3, p.301]). Throughout this paper $A(X)$ will always denote a complete ring of functions.

We first remark that if $f \in A(X)$, then $|f| \in A(X)$. Thus $A(X)$ is a lattice, since $f \vee g=(f+g+|f-g|) / 2$ and $f \wedge g=(f+g-|f-g|) / 2$. (See Willard [8, p.291].)

LEMMA 1.1. If $f \in A(X)$ and $0<c \leqslant f(x)$ for all $x \in X$, then $f$ is invertible in $A(X)$.

Proof: Choose $a>0$ such that $0<a c \leqslant a f(x)<1$ for all $x \in X$. Then $0<1-a f(x) \leqslant 1-a c<1$, so

$$
\frac{1}{a f(x)}=\sum_{n=0}^{\infty}(1-a f(x))^{n} \leqslant \sum_{n=0}^{\infty}(1-a c)^{n}<\infty
$$

Since $A(X)$ is uniformly closed, $[a f]^{-1} \in A(X)$, and hence $f^{-1} \in A(X)$.

For $f \in C(X)$, we write $Z(f)$ for the zero set of $f$; that is, $Z(f)=\{x \in X: f(x)=$ $0\}$. We denote the collection of all zero sets in $X$ by $Z[X]$. A cozero set is the complement of a zero set. For a cozero set $E$ we say that $f \in A(X)$ is $E$-regular if 
there exists $g \in A(X)$ such that $\left.f g\right|_{E} \equiv 1$; thus, in a sense, $f$ is 'locally invertible' on $E$.

LEMмa 1.2. Let $f, g \in A(X)$ and let $E, F$ be cozero sets in $X$.

(a) If $f$ is $E$-regular and $F \subseteq E$, then $f$ is $F$-regular.

(b) If $f$ is $E$-regular and $F$-regular, then $f$ is $E \cup F$-regular.

(c) If $f(x) \geqslant c>0$ for all $x \in E$, then $f$ is $E$-regular.

(d) If $0<f(x) \leqslant g(x)$ for all $x \in E$ and if $f$ is $E$-regular, then $g$ is $E$-regular.

(e) If $f$ is $E$-regular and $g$ is $F$-regular, then $f g$ is $E \cap F$-regular and $f^{2}+g^{2}$ is $E \cup F$-regular.

(f) $f$ is $E$-regular if and only if $|f|$ is $E$-regular.

Proof: The proofs of (a), (b), (d) and (e) are identical to the corresponding parts of $[6$, Lemma 1$]$, so we prove (c) and (f).

(c) Let $h=c \vee f$. Then $h \in A(X)$, and so $h^{-1} \in A(X)$ by Lemma 1.1. The result follows since $\left.h^{-1} f\right|_{E}=1$.

(f) If $f$ is $E$-regular then there exists $g \in A(X)$ such that $\left.f g\right|_{E}=1$. But then $\left.|f||g|\right|_{E}=1$, so since $|f|,|g| \in A(X),|f|$ is $E$-regular. Conversely, if $|f|$ is $E$-regular, there exists $g \in A(X)$ such that $\left.g|f|\right|_{E}=1$. Let $E_{1}=\{x \in E: f(x)>0\}$ and let $E_{2}=\{x \in E: f(x)<0\}$. Then $\left.f g\right|_{E_{1}}=1$ and $\left.f(-g)\right|_{E_{2}}=1$, and so by (b), $f$ is $E_{1} \cup E_{2}$-regular (that is, $E$-regular).

As in [6], for $f \in A(X)$ we define $Z_{A}(f)=\left\{E \in Z[X]: f\right.$ is $E^{\text {c-regular }}$; for $J$ an ideal of $A(X)$ we write $Z_{A}[J]=\bigcup_{f \in J} Z_{A}(f)$; and for $\mathcal{F}$ a $z$-filter on $X$ we write $Z_{A}^{\leftarrow}[\mathcal{F}]=\left\{f \in A(X): Z_{A}(f) \subseteq \mathcal{F}\right\}$. With these definitions, the following theorem can be proved as in [6, Theorems 1, 2 and 3].

THEOREM 1.3 .

(a) The function $f \in A(X)$ is noninvertible in $A(X)$ if and only if $z_{A}(f)$ is a $z$-filter on $X$.

(b) If $I$ is an ideal in $A(X)$, then $Z_{A}[I]$ is a $z$-filter on $X$.

(c) If $\mathcal{F}$ is a $z$-filter on $X$, then $Z_{A}^{\leftarrow}[\mathcal{F}]$ is an ideal in $A(X)$.

The next theorem gives a more explicit description of the ideal $Z_{A}^{\leftarrow}[\mathcal{F}]$.

Theorem 1.4. If $\mathcal{F}$ is any $z$-filter on $X$, then $Z_{A}^{\leftarrow}[\mathcal{F}]=\{f \in A(X)$ : $\left.\lim _{\mathcal{F}} f=0\right\}$.

Proof: Let $f \in Z_{A}^{\leftarrow}[\mathcal{F}]$. This means $Z_{A}(f) \subseteq \mathcal{F}$. We claim that $\lim _{\mathcal{Z}(f)} f=0$. To prove this we shall show that $\mathcal{F}_{0}$, the $z$-filter of zero-set neighbourhoods of 0 in $\mathbb{R}$, is contained in $\mathcal{B}=\{f(E): E \in Z(f)\}$. Let $V \in \mathcal{F}_{\mathbf{0}}$ and let $E=f^{-1}(V)$. 
Then $E \in Z(|f|)$ by Lemma $1.2(\mathrm{c})$, and hence $E \in Z(f)$ by Lemma $1.2(\mathrm{f})$. Thus $V=f(E) \in \mathcal{F}_{0}$, so that $\mathcal{F}_{0} \subset \mathcal{B}$. This proves the claim. It follows that $\lim _{\mathcal{F}} f=0$.

Conversely, suppose $f \in A(X)$ with $\lim _{\mathcal{F}} f=0$. Then clearly $\lim _{\mathcal{F}} f h=0$ for all $h \in A(X)$ (since every $h \in A(X)$ is bounded). We claim that for every $E \in \mathcal{Z}(f)$ there exists $F \in \mathcal{F}$ such that $F \subseteq E$. For it not, we have that $F \cap E^{c} \neq \emptyset$ for every $F \in \mathcal{F}$. Choosing $h \in A(X)$ such that $\left.f h\right|_{E^{c}} \equiv 1$ would then show that 1 is a cluster point of $\{f h(F): F \in \mathcal{F}\}$, a contradiction. Thus $\mathcal{F} \supseteq \mathcal{Z}(f)$, and so $f \in Z_{A}^{-}[\mathcal{F}]$.

We now show that $Z_{A}^{\leftarrow}$ maps $z$-ultrafilters to maximal ideals. We need a preliminary lemma.

LEMмA 1.5. Let $\mathcal{U}$ be a $z$-ultrafilter on $X$. Then $Z_{A}^{\leftarrow}[\mathcal{U}]=A(X) \cap Z_{C^{*}}^{\leftarrow}[\mathcal{U}]$.

Proof: We claim that for every $f \in A(X), Z_{A}(f)=Z_{C^{*}}(f)$. For clearly $z_{A}(f) \subseteq$ $Z_{C^{*}}(f)$. So let $E \in Z_{C^{*}}(f)$, and choose $g \in C^{*}(X)$ such that $\left.f g\right|_{E}=1$. Since $g$ is bounded, there exists $c>0$ such that $|f(x)| \geqslant c$ for all $x \in E$. Thus by Lemma 1.2(c) and (f), $f$ is $E^{c}$-regular in $A(X)$; that is, $E \in Z_{A}(f)$. This proves the claim.

Now let $f \in Z_{A}^{\leftarrow}[\mathcal{U}]$. Then $Z_{A}(f) \subset \mathcal{U}$, so by the claim, $Z_{C^{*}}(f) \subset \mathcal{U}$. Thus $f \in$ $Z_{C^{*}}^{\leftarrow}[\mathcal{U}]$, and hence $Z_{A}^{\leftarrow}[\mathcal{U}] \subseteq A(X) \cap Z_{C^{*}}[\mathcal{U}]$. Conversely, suppose $f \in A(X) \cap Z_{C^{*}}^{\leftarrow}[\mathcal{U}]$. Then $Z_{C^{*}}(f) \subset \mathcal{U}$, and so $Z_{A}(f) \subset \mathcal{U}$, whence $f \in Z_{A}^{\leftarrow}[\mathcal{U}]$.

It follows from the first part of the proof of the preceding lemma that $Z(f)=$ $\cap Z_{A}(f)$.

THEOREM 1.6. If $\mathcal{U}$ is a $z$-ultrafilter on $X$, then $\mathcal{Z}_{A}^{\leftarrow}[\mathcal{U}]$ is a maximal ideal in $A(X)$.

Proof: Let $M=Z_{C^{*}}^{\leftarrow}[\mathcal{U}]$ and let $N=Z_{A}^{\leftarrow}[\mathcal{U}]$. By Lemma 1.5 there is a natural embedding $i: A(X) / N \rightarrow C^{*}(X) / M$, and by [2, Theorem 3.2(b)], $M$ is a maximal ideal of $C^{*}(X)$, so there exists an isomorphism $J: C^{*}(X) / M \rightarrow \mathbb{R}$. The map $k: \mathbb{R} \rightarrow$ $A(X) / N$ defined by $r \mapsto r+N$ is clearly one-to-one. Now consider the map $\phi=j \circ i \circ k$, $\phi: \mathbb{R} \rightarrow \mathbb{R}$. Since $\phi$ is an embedding, it must be the identity map on $\mathbb{R}$, and so $A(X) / N \simeq \mathbb{R}$. Thus $N$ is a maximal ideal in $A(X)$.

THEOREM 1.7. There is a surjection from the set of $z$-ultrafilters on $X$ onto the set of maximal ideals of $A(X)$ given by

$$
\mathcal{U} \mapsto Z_{A}^{\leftarrow}[\mathcal{U}]
$$

Proof: Theorem 1.6 guarantees the existence of this map. To show it is onto, let $M$ be a maximal ideal in $A(X)$, and let $\mathcal{U}$ be a $z$-ultra filter containing the $z$-filter $\mathcal{Z}_{A}[M]$. Then $M \subseteq \mathcal{Z}_{A}^{\leftarrow}[\mathcal{U}]$, and so $M=Z_{\leftarrow}^{\leftarrow}[\mathcal{U}]$.

EXAMPLE 1.8: We show that the map defined in the preceding theorem need not be one-to-one. Let $X=(0,1)$, and let $A(X)=\left\{f \in C^{*}(X): \lim _{x \rightarrow 0^{+}} f(x)=\lim _{x \rightarrow 1^{-}} f(x)\right\}$. 
Clearly $A(X)$ is a complete ring of functions. Let $\mathcal{U}_{0}$ and $\mathcal{U}_{1}$ denote the $z$-ultrafilters on $X$ that converge (in $\beta X$ ) to 0 and 1 respectively. Clearly $\mathcal{U}_{0}$ and $\mathcal{U}_{1}$ are distinct. However, by Theorem 1.4 we have

$$
Z_{A}^{-}\left[\mathcal{U}_{0}\right]=\left\{f \in A(X): \lim _{\mathcal{U}_{0}} f=0\right\}=\left\{f \in A(X): \lim _{\mathcal{U}_{1}} f=0\right\}=Z_{A}^{\leftarrow}\left[\mathcal{U}_{1}\right]
$$

\section{THE CORRESPONDENCE BETWEEN COMPACTIFICATIONS AND SUBRINGS}

In this section we show that there is a natural correspondence between complete subrings of $C^{*}(X)$ and compactifications of $X$. It is well known that every compactification of $X$ is a topological quotient of $\beta X$. Our map $z_{A}^{\leftarrow}$ gives an explicit construction of the equivalence relation on $\beta X$ leading to each such quotient.

Recall that in the Stone construction of $\beta X$, the points of $\beta X$ are the $z$-ultrafilters on $X$. The points of $\beta X$ will be denoted by the letters $p, q, \ldots$ When we need to emphasise that these points are $z$-ultrafilters, we shall write $\mathcal{U}_{p}, \mathcal{U}_{q}, \ldots$ In this notation the space $X$ is canonically embedded in $\beta X$ via the map $p \mapsto \mathcal{U}_{p}$, where $\mathcal{U}_{p}$ is the $z$-ultrafilter of all zero sets in $X$ containing $p \in X$.

DEFINITION: Let $A(X)$ be a complete ring of functions. We define an equivalence relation $\tilde{A}_{\boldsymbol{A}}$ on $\beta X$ as follows:

$$
p \underset{A}{\sim} q \text { if and only if } Z_{A}^{\leftarrow}\left[\mathcal{U}_{p}\right]=Z_{A}^{\leftarrow}\left[\mathcal{U}_{q}\right]
$$

This relation does not identify points of $X$, since if $p \neq q \in X$ there exists $f \in A(X)$ such that $f(x)=0, f(y) \neq 0$, and so $Z_{A}^{\leftarrow}\left[\mathcal{U}_{p}\right] \neq Z_{A}^{\leftarrow}\left[\mathcal{U}_{q}\right]$. Thus $\beta X / \sim_{A}$ is a compactification of $X$. We denote it by $\beta_{A} X$.

In the sequel, we write $M_{p}$ for the maximal ideal $z_{A}^{\leftarrow}\left[\mathcal{U}_{p}\right]$; thus $p_{A}^{\sim} q$ if and only if $M_{p}=M_{q}$. For $f \in C^{*}(X)$ we denote its extension to $\beta X$ by $f^{\beta}$, and so $f^{\beta}(p)=\lim _{\mathcal{U}_{p}} f$ for all $p \in \beta X$. We also write $A(\beta X)$ for the subalgebra of $C^{*}(\beta X)$ consisting of the extensions to $\beta X$ of the functions in $A(X)$; thus $A(\beta X)=\left\{f^{\beta}: f \in A(X)\right\}$.

Lemma 2.1. $M_{p}=M_{q}$ if and only if $f^{\beta}(p)=f^{\beta}(q)$ for all $f \in A(X)$.

Proof: It follows immediately from Theorem 1.4 and the preceding discussion that $M_{p}=\left\{f \in A(X): f^{\mathcal{\beta}}(p)=0\right\}$. So if $p \underset{A}{\sim} q$ then $f^{\beta}(p)=0$ if and only if $f^{\beta}(q)=0$ for all $f \in A(X)$.

Now suppose $M_{p}=M_{q}$, and let $f \in A(X)$, with $f^{\beta}(p)=r \in \mathbb{R}$. Then $(f-r)^{\beta}(p)=0$, and hence $(f-r)^{\beta}(q)=0$, which means that $f^{\beta}(q)=r$. Thus $f^{\beta}(p)=f^{\beta}(q)$ for all $f \in A(X)$. The converse is obvious.

The next theorem makes clear that the situation described in Example 1.8 is in fact the typical case. 
Theorem 2.2. $Z_{A}^{\leftarrow}$ is one-to-one on $\beta X$ if and only if $A(X)=C^{*}(X)$.

Proof: The subring $A(\beta X)$ of $C^{*}(\beta X)$ is clearly uniformly closed, contains the constants, and is naturally isomorphic to $A(X)$. Now suppose $Z_{A}^{\leftarrow}$ is one-to-one, and let $p \neq q \in \beta X$. Then by Lemma 2.1, there exists $f \in A(X)$ such that $f^{\beta}(p) \neq f^{\beta}(q)$. Thus $A(\beta X)$ separates the points of $\beta X$, and so by the Stone-Weierstrass Theorem, $A(\beta X)=C^{*}(\beta X) \simeq C^{*}(X)$. Hence $A(X)=C^{*}(X)$. The converse is known $[6,2]$. $]$

Theorem 2.3: Let $f \in C^{*}(X)$. Then $f$ has an extension $f^{A}$ to $\beta_{A} X$ if and only if $f \in A(X)$.

Proof: Let $C_{A}(X)=\left\{f \in C^{*}(X): f\right.$ has an extension $f^{A}$ to $\left.\beta_{A} X\right\}$, and let $\eta_{A}: \beta X \rightarrow \beta_{A} X$ denote the canonical projection. We must show that $C_{A}(X)=A(X)$. So let $f \in A(X)$, and suppose $p, q \in \beta X$ with $p \sim_{A} q$. Then $M^{p}=M^{q}$, and so by Lemma 2.1, $f^{\beta}(p)=f^{\beta}(q)$; that is, $f^{\beta}$ is constant on the equivalence classes modulo $\widetilde{A}$. Thus there exists a unique map $f^{A}: \beta_{A} X \rightarrow \mathbb{R}$ such that $f^{\beta}=f^{A} \circ \eta_{A}$, and clearly $f^{A}$ is an extension of $f$. This means that $f \in C_{A}(X)$, and so $A(X) \subseteq C_{A}(X)$.

Clearly the map $\varphi: C_{A}(X) \rightarrow C^{*}\left(\beta_{A} X\right)$ defined by $f \mapsto f^{A}$ is an embedding, natural in the sense that $\left.f\right|_{X}=\left.\varphi(f)\right|_{X}$. The subalgebra $\varphi(A(X))$ of $C^{*}\left(\beta_{A} X\right)$ contains the constants and is uniformly closed (since $A(X)$ is). Moreover, it separates the points of $\beta_{A} X$, since if $p, q \in \beta X$ lie in different equivalence classes $[p],[q] \in \beta_{A} X$, then by Lemma 2.1 there exists $f \in A(X)$ such that $f^{\beta}(p) \neq f^{\beta}(q)$, and so $f^{A}([p]) \neq f^{A}([q])$. Thus by the Stone-Weierstrass Theorem, $\varphi(A(X))=C^{*}\left(\beta_{A} X\right)$, and so $A(X)=C_{A}(X)$.

Theorem 2.3 is a generalisation of the universal property of the Stone-Čech compactification. For if $A(X)$ is taken to be all of $C^{*}(X)$, Theorem 2.3 says: a continuous real-valued function on $X$ can be extended to $\beta X$ if and only if $f$ is bounded.

For every complete ring of functions we have constructed a compactification of $X$. We now show that every compactification arises in this fashion, and that the resulting correspondence between rings and compactifications is one-to-one.

DEFINITION: Let $X$ be a complete regular space and let $\alpha X$ be a compactification of $X$. We denote by $A_{\alpha}(X)$ the subring of $C^{*}(X)$ consisting of those functions that extend to $\alpha X$. We write $f^{\alpha}$ for the extension of $f$ to $\alpha X$; thus:

$$
A_{\alpha}(X)=\left\{f \in C^{*}(X): f \text { has an extension } f^{\alpha}\right\}
$$

Proposition 2.4. $A_{\alpha}(X)$ is a complete ring of functions.

Proof: Clearly $A_{\alpha}(X)$ is a subring of $C^{*}(X)$ that contains the constants and is uniformly closed. We show that it separates points and closed sets. Let $B$ be a closed subset of $X$ and let $x \in X, x \notin B$. Denoting the closure operator in $\alpha X$ by $\mathrm{cl}_{\alpha}$, we 
have $x \notin \operatorname{cl}_{\alpha} B$, so there exists $f \in C^{*}(\alpha X)$ such that $f(x) \notin \operatorname{cl}_{\mathbb{R}}\left(f\left(\mathrm{cl}_{\alpha} B\right)\right)$. Hence $\left.f\right|_{X}$ separates $x$ and $B$.

Recall that two compactifications of $X$ are equivalent if there is a homeomorphism between them that leaves $X$ pointwise fixed.

THEOREM 2.5. There is a one-to-one correspondence between the complete subrings of $C^{*}(X)$ and the compactifications of $X$.

Proof: We claim that the compactification $\alpha X$ is equivalent to the compactification $\beta_{A_{\alpha}} X$. To prove this, we first note that $A_{\alpha}(X)$ is isomorphic to $C^{*}(\alpha X)$, since $A_{\alpha}(X)$ consists precisely of those functions in $C^{*}(X)$ that extend (necessarily uniquely) to $\alpha X$. This induces a homeomorphism between the compact spaces $\beta_{A_{\alpha}} X$ and $\beta_{C^{*}(\alpha X)} \alpha X$ that leaves $X$ fixed. But $\beta_{C^{*}(\alpha X)} \alpha X=\beta(\alpha X)=\alpha X$, since $\alpha X$ is compact. This proves the claim.

It follows from Theorem 2.3 that if $A(X)$ is a complete ring of functions then $A_{\beta_{A}}(X)=A(X)$. Thus we have constructed two correspondences, one taking complete rings of functions on $X$ to compactifications of $X$,

$$
A(X) \mapsto \beta_{A} X,
$$

and one taking compactifications to complete rings,

$$
\alpha X \mapsto A_{\alpha}(X) .
$$

We have also shown that these correspondences are inverse to each other, and so they are one-to-one and onto.

\section{INTERSECTIONS OF FREE IDEALS}

An ideal $I$ in $A(X)$ is free if $\bigcap_{f \in I} z_{A}(f)=\emptyset$; otherwise it is fixed. In view of the remark following Lemma 1.5, an ideal $I$ is free or fixed according to whether $\bigcap_{f \in I} Z(f)$ is empty or not. The problem of describing the intersection of the free maximal ideals in $C(X)$ has been studied extensively (see, for example, [4, p.123], [5], and [7]). The analogous problem for subrings of $C(X)$ containing $C^{*}(X)$ was considered in [6] and [2]. In this section we describe the intersections of the free maximal ideals and of all free ideals in subrings $A(X)$ of $C^{*}(X)$.

LEMMA 3.1. The ideal $M$ in $A(X)$ is maximal if and only if $M=A(X) \cap N$, where $N$ is a maximal ideal in $C^{*}(X)$.

Proof: If $N$ is maximal in $C^{*}(X)$, then there is a $z$-ultrafilter $\mathcal{U}$ on $X$ such that $N=Z_{C^{*}}[\mathcal{U}]$, by Theorem 1.7. But then $M=A(X) \cap N=A(X) \cap z_{C^{*}}^{-}[\mathcal{U}]=Z_{A}^{\leftarrow}[\mathcal{U}]$, 
which is maximal (Lemma 1.5 and Theorem 1.6). Conversely, if $M$ is maximal in $A(X)$, then $M=Z_{A}^{\leftarrow}[\mathcal{U}]$ for some $z$-ultrafilter on $X$. Thus we have $M=A(X) \cap N$, where $N=Z_{C^{*}}^{\leftarrow}[\mathcal{U}]$ is maximal in $C^{*}(X)$.

In particular, $M$ is a free maximal ideal in $A(X)$ if and only if $M=A(X) \cap N$ for some free maximal ideal $N$ in $C^{*}(X)$. Following [4], we write $C_{\infty}(X)$ for the subalgebra of $C^{*}(X)$ consisting of the functions that vanish at infinity, where a function $f \in C^{*}(X)$ is said to vanish at infinity if for every $n \in \mathbb{N}$ there is a compact subset $K$ of $X$ such that $|f(x)| \leqslant 1 / n$ for all $x \in X \backslash K$.

THEOREM 3.2. The intersection of the free maximal ideals in $A(X)$ is $C_{\infty}(X) \cap$ $A(X)$.

Proof: This follows immediately from Lemma 3.1 and [4, p.109].

If $X$ is locally compact, then it turns out that the intersection of the free maximal ideals in any $A(X)$ is simply $C_{\infty}(X)$, since $C_{\infty}(X) \subset A(X)$ in this case, as we now show. We will require some preliminary results. In the sequel, $C_{K}(X)$ denotes the ring of continuous real-valued functions of compact support on $X$.

LEMMA 3.3. The completely regular space $X$ is locally compact if and only if $C_{K}(X)$ separates points and closed sets in $X$.

Proof: That $C_{K}(X)$ has this property if $X$ is locally compact follows from [3, Corollary 3.3.3]. So suppose that $C_{K}(X)$ separates points and closed sets. Let $x \in X$, let $V$ be any open neighbourhood of $x$, and let $D=V^{c}$. Choose an $f \in C_{K}(X)$ that separates $x$ and $D$, with $f(x)>0$, and let $I=(a, b)$, where $0<a<f(x)<b$ and $\bar{I} \cap \overline{f(D)}=\emptyset$. Then $\overline{f^{-1}(I)}$ is a compact neighbourhood of $x$ since it is contained in the support of $f$.

Proposition 3.4 .

(a) If $A(X) \subseteq B(X)$ are complete rings of functions, then there exists a continuous projection $\pi: \beta_{B} X \rightarrow \beta_{A} X$ such that $\left.\pi\right|_{X}$ is the identity on $X$.

(b) if $\alpha X$ and $\gamma X$ are two compactifications of $X$ and if there exists a continuous projection $\pi: \alpha X \rightarrow \gamma X$ such that $\left.\pi\right|_{X}$ is the identity on $X$, then $A_{\alpha}(X) \supseteq A_{\gamma}(X)$.

Proof: This follows from Lemma 2.1 and the definitions of the concepts involved.

THEOREM 3.5. If $X$ is locally compact, then every complete ring of functions $A(X)$ contains $C_{\infty}(X)$.

Proof: Let $X$ be locally compact. We claim that $C_{\infty}(X)$ is uniformly closed. For suppose $f_{k} \rightarrow f$ uniformly, with $f_{k} \in C_{\infty}(X)$. Let $n \in \mathbb{N}$ and choose $N$ such 
that $\left|f_{k}-f\right|<1 /(2 n)$ for all $k \geqslant N$. Choose a compact set $K \subset X$ such that $f_{N}(x) \leqslant 1 /(2 n)$ for all $x \in X \backslash K$. Then $|f(x)| \leqslant\left|f(x)-f_{N}(x)\right|+\left|f_{N}(x)\right| \leqslant 1 / n$ for all $x \in X \backslash K$.

Let $L(X)$ be the subring of $C^{*}(X)$ generated by $C_{\infty}(X)$ together with the constant functions. By the preceding claim and Lemma 3.3, $L(X)$ is a complete ring of functions (since $C_{K}(X) \subseteq C_{\infty}(X)$ ). The compactification $\beta_{L} X$ is clearly the onepoint compactification of $X$, which is a quotient of every compactification. Thus by Proposition 3.4, every complete ring of functions on $X$ contains $L(X)$.

Corollary 3.6. If $X$ is locally compact, then the intersection of the free maximal ideals in any complete ring of functions $A(X)$ is $C_{\infty}(X)$.

We now consider the intersection of all free ideals in $A(X)$. For every $p \in \beta X$ we define $\mathcal{Q}_{A}^{p}=\left\{f \in A(X): p \in\right.$ int $\left.Z_{\beta X}\left(f^{\beta}\right)\right\}$. This is clearly an ideal in $A(X)$.

Lemma 3.7. $\mathcal{Q}_{A}^{p}$ is free for all $p \in \beta X \backslash X$.

Proof: Let $p \in \beta X \backslash X$. We claim that for every $x \in X$ there exists $f \in \mathcal{Q}_{A}^{p}$ such that $f(x) \neq 0$. The lemma follows, since we then have $\bigcap_{f \in \mathcal{Q}_{A}^{p}} Z_{A}(f)=\bigcap_{f \in \mathcal{Q}_{A}^{p}} Z(f)=\emptyset$. To prove the claim, choose a closed $\beta X$-neighbourhood $F$ of $p$ such that $x \notin F$. Now $F \cap X$ is a closed nonempty set in $X$, so there exists $f \in A(X)$ such that $f(F \cap X)=0$ and $f(x)=1$. Clearly $f^{\beta}(F)=0$, so $f \in \mathcal{Q}_{A}^{p}$.

It can be shown that an ideal in $A(X)$ is free if and only if for every compact set $K \subset X$ there exists $f \in I$ such that $Z(f) \cap K=\emptyset$ (see [2, Lemma 5.2], whose proof applies also in our situation here). In addition, we have the following characterisation of $C_{K}(X)$ :

$$
C_{K}(X)=\left\{f \in C^{*}(X): Z_{\beta X}\left(f^{\beta}\right) \text { is a neighbourhood of } \beta X \backslash X\right\}
$$

(see $[4$, p.109]).

THEOREM 3.8. The intersection of the free ideals in any complete ring of functions $A(X)$ is $C_{K}(X) \cap A(X)$.

Proof: Let $f \in C_{K}(X) \cap A(X)$ and let $I$ be a free ideal in $A(X)$. We write $E=\overline{(X \backslash Z(f))}$ for the support of $f$. By the remarks preceding this theorem there exists $g \in I$ such that $Z(g) \cap E=\emptyset$. Let $c$ be the minimum value of $|g|$ on $E$. Clearly $c>0$, so by Lemma $1.2(\mathrm{c})$ and (f), $g$ is $E$-regular. Thus there exists $h \in A(X)$ such that $\left.g h\right|_{E} \equiv 1$. Thus $f=f g h \in I$.

Conversely, suppose $f$ is in every free ideal. Then $f \in \mathcal{Q}_{A}^{p}$ for every $p \in \beta X \backslash X$ by Lemma 3.7, and so $\beta X \backslash X \subseteq$ int $Z_{\beta X}\left(f^{\beta}\right)$. Thus $f \in C_{K}(X)$.

Comparing Theorems 3.5 and 3.8 , we have the following corollary. 
COROLLARY 3.9. If $X$ is locally compact then the intersection of the free ideals in any complete ring of functions $A(X)$ is $C_{K}(X)$.

\section{REFERENCES}

[1] C.E. Aull, Rings of continuous functions (Marcel Dekker, New York, 1985).

[2] H.L. Byun and S. Watson, 'Prime and maximal ideals in subrings of $C(X)$ ', Topology Appl. 40 (1991), 45-62.

[3] R. Engelking, General topology (PWN-Polish Scientific Publishers, Warsaw, 1977).

[4] L. Gillman and M. Jerison, Rings of continuous functions (Springer-Verlag, Berlin, Heidelberg, New York, 1978).

[5] M. Mandelker, 'Supports of continuous functions', Trans. Amer. Math. Soc. 156 (1971), 73-83.

[6] L. Redlin and S. Watson, 'Maximal ideals in subalgebras of $C(X)$ ', Proc. Amer. Math. Soc. 100 (1987), 763-766.

[7] S.M. Robinson, 'A note on the intersection of free maximal ideals', J. Austral. Math. Soc. 10 (1969), 204-206.

[8] S. Willard, General topology (Addison-Wesley, Reading, Mass., 1970).

Department of Mathematics

California State University

Long Beach, CA 90840

United States of America

Department of Mathematics

California State University

Long Beach, CA 90840

United States of America
Department of Mathematics

The Pennsylvania State University

Abington, PA 19001

United States of America 